\title{
Procesos de esencialización de hombres y mujeres: Un estudio comparado Brasil-España
}

\author{
1 Universidade Federal da Babia (Brasil). \\ 2 Universidad Complutense de Madrid (España).
}

Marcos-Emanoel Pereira ${ }^{1 *}$, José-Luis Álvaro ${ }^{2}$ y Alicia Garrido ${ }^{2}$

\begin{abstract}
Resumen: En esta investigación se presentan los resultados de un experimento, fundamentado en el paradigma del trasplante de cerebros, realizado en Brasil y España y cuya finalidad fue la de estudiar diversos procesos de esencialización de diferentes categorías sociales. Fue posible identificar que las categorías sociales naturalizables son más esencializadas que las entitativas, que la esencialización es más intensa en Brasil que en España y que la dirección del supuesto trasplante de cerebros en la que se basa el experimento realizado tuvo impacto sólo entre los participantes españoles. En ambos países la atribución de causas internas fue la justificación más utilizada, siendo especialmente destacable una tendencia a la biologización del género, con referencias a conceptos como los de hormonas o genes, utilizados como argumentos para la elaboración de explicaciones de sentido común del comportamiento humano. En conjunto, los resultados encontrados permiten concluir que la esencialización cumple un papel importante en la perpetuación del sexismo y de otras formas de estereotipación de las diferencias sexuales.

Palabras clave: Género; estereotipos; esencialización; natularización; enti tatividad.
\end{abstract}

\section{Introducción}

Si hasta finales del siglo XIX e inicios del siglo XX, el criterio fundamental para la categorización, fundamentada en las diferencias entre sexos, implicaba básicamente la utilización de criterios biológicos, a mediados del siglo XX emerge un movimiento de crítica a esta naturalización basado en la idea de que las relaciones de género dependen más del impacto de factores sociales y políticos que de elementos ligados a una supuesta naturaleza humana (Fine, 2010). Las transformaciones sociales y los profundos cambios en las costumbres, iniciados a partir de la década de los sesenta del siglo pasado, se reflejaron en los estudios de género, que se volcaron en la crítica a las versiones androcéntricas de la ciencia, en la medida en que no contribuían a fomentar las transformaciones necesarias para lograr la igualdad entre hombres y mujeres. En este sentido, uno de los objetivos fundamentales de las epistemologías feministas fue demostrar los límites de las epistemologías clásicas, mediante el cuestionamiento del saber masculino como criterio universal de validación del conocimiento (Anderson, 1995; Duran, 1991). La aparición de estudios científicos influidos por esta nueva epistemología feminista favoreció el auge de debates relacionados con el problema de las diferencias entre géneros, centrándose principalmente en establecer una distinción entre los conceptos de sexo y género, relacionando el primero con la naturaleza y el segundo con la cultura. Pese a todo, e involuntariamente, esta distinción acabó por reproducir la vieja dicotomía entre aquellas clases susceptibles de ser clasificadas

* Dirección para correspondencia [Correspondence address]:

Marcos Emanoel Pereira. Universidade Federal da Bahia. Instituto de Psicologia. Campus Universitário São Lázaro. 41940-220 - Salvador, BA (Brasil). E-mail: memanoel@gmail.com
Title: Essentialization processes in men and women: A Brazil-Spain comparative study.

Abstract: The main objective of this research is to present the results of an experiment based in the brain transplant paradigm. It was done in Brazil and Spain and its aim was to study various essentialization processes among different social categories. It was possible to identify that naturalizable social categories are more essentialized than entitative ones, that essencialization is greater in Brazil than in Spain, and that the direction of the supposed brain transplant in which the experiment was based had an impact only among Spanish participants. In both countries the attribution of internal causes was the most common explanation together with a biologization of gender with special reference to hormones and genes used as arguments to elaborate common sense explanations of human behavior. $\mathrm{Al}$ together the results found lead us to the conclusion than essentialization plays an important role in the perpetuation of sexism and other forms of gender roles stereotypes.

Key words: Gender; stereotypes; essentilization; naturalization; entitativity.

por su pertenencia a la naturaleza y aquellas otras pertenecientes al ámbito de la cultura, en la medida en que delimitaba el concepto de sexo a lo biológicamente determinado y, por tanto, naturalizable, y consideraba al género como resultado de una práctica socio-cultural, y, por tanto, como una construcción social determinada por convenciones sociales.

Paradójicamente, esta diferenciación surgida como una forma estratégica de combatir aquellas prácticas consideradas como androcéntricas acabo por ofrecer un refugio a la naturalización y el pensamiento esencialista. La exigencia de igualdad de derechos, aliada a los esfuerzos por retirar cualquier connotación de naturaleza biológica en los discursos acerca de las diferencias de género, acabó, inintencionadamente, por reforzar el discurso sobre las diferencias entre sexos. Aunque la esencialización y la naturalización no se apliquen más al ámbito de las relaciones de género, definidas en términos de la clase masculino-femenino, posibilita, en cierta forma, el establecimiento de diferencias entre sexos, definidos por la clase hombre-mujer, lo cual escapa al ámbito cultural definido por la clase masculino-femenino y da pie a la naturalización y la esencialización, reforzando así los estereotipos de género.

\section{Estereotipos, categorización social, esencialismo psicológico y teorías explicativas}

Los estereotipos son creencias sociales compartidas sobre determinados grupos, agregados y categorías sociales. Estas creencias se refieren a similitudes en la apariencia física o en los patrones de conducta y se fundamentan en teorías basadas en explicaciones sobre el contenido de dichos atributos. En el plano práctico, los estereotipos cumplen dos funciones, una relacionada con la economía psíquica, al 
permitir simplificar la realidad social, y otra que consiste en mantener el estatus quo, al ofrecer los medios y los recursos necesarios para la creación y la promoción de estrategias destinadas a justificar y racionalizar las diferencias sociales (Pereira, 2002; Pereira, Modesto y Matos, 2013).

Allport (1962) ya evidenció el papel ejercido por la inclusión de individuos en clases para la formación de estereotipos sociales al sugerir que la categorización social debería ser considerada como un proceso mediante el cual una persona dejaría de ser percibida como individuo con características singulares para ser percibido y juzgado como miembro de un grupo o categoría social. La percepción de homogeneidad entre los miembros de una categoría desempeña un papel decisivo en este proceso. El pensamiento categórico, sin embargo, no se aplica de igual forma a todas las categorías sociales. Algunas, como el género, la edad o la raza se diferencian de las demás porque no son escogidas por los individuos, sino porque su inclusión en las mismas depende de argumentos basados en teorías y conceptos biologicistas o genetistas, lo cual genera un potencial inductivo que permite la rápida inclusión de un miembro en una categoría, haciendo que sean más fácilmente naturalizables. En el pensamiento esencialista el proceso de categorización si bien comparte el supuesto de que éste depende de las similitudes superficiales identificadas en las apariencias de los miembros de la categoría, añade la creencia de que los miembros de un grupo comparten características comunes más profundas que les diferencian de los miembros de otras categorías sociales (Gelman y Wellman, 1991), lo cual permite asumir la existencia de una esencia para cada categoría. De acuerdo con Medin (1989), esto representa una diferencia fundamental en relación a las teorías clásicas de la categorización, ya que éstas se basan en la idea de que la percepción de similitudes o de homogeneidad es la causa de la categorización, mientras que el esencialismo postula que la semejanza entre los individuos pertenecientes a una categoría es una consecuencia de la aplicación de una teoría explicativa que permite agrupar a los individuos como semejantes, aunque dicha semejanza no se manifieste en su apariencia física.

Los primeros estudios psicosociales sobre el esencialismo se preocuparon por analizar aquellas categorías naturalizables diferenciándolas de otras categorías sociales, en la medida en que la categorización de las primeras era realizada mediante criterios de clasificación de índole biológica. La aplicación a categorías sociales de criterios basados en la categorización natural es lo que llevaría a su esencialización (Rothbart y Taylor, 1992).

Una cuestión importante, inherente a la teoría sobre el pensamiento esencialista, es la relativa a qué es lo que hace que unos grupos sean más fácilmente esencializados que otros. La respuesta a esta pregunta pasa por entender que la esencialización no ocurre exclusivamente a través de la naturalización, sino que también debe incluir una segunda dimensión que es la entitatividad (Yzerbyt, Corneille y Estrada, 2001). Autores como Demoulin, Leyens e Yzerbyt (2006) muestran que la percepción de una categoría como naturali- zable o entitativa implica, en ambos supuestos, procesos de esencialización. La esencialización, por tanto, puede ser predicha como una consecuencia de la categorización, independientemente de que está lleva a considerar un grupo como naturalizable o entitativo. La naturalización incluye características como la inmutabilidad, la invariabilidad histórica y la existencia de límites firmemente establecidos entre las clases, lo que posibilita la categorización de los grupos a partir de dimensiones como la edad, la raza y el género. Además de esta primera dimensión clásica, es posible establecer otra modalidad de esencialización, a través de la dimensión entitativa. De acuerdo con Campbell (1958), dos factores son decisivos en este proceso de transformación de agregados humanos en entidades diferenciadas: en primer lugar, la semejanza y proximidad entre los miembros de una categoría $y$, en segundo lugar, su estructura organizacional. De esta forma, se desarrolla una concepción reificada de los grupos sociales, que pasan a ser percibidos como entidades homogéneas cuyos miembros comparten una identidad particular y común, fundamentada en sus propiedades inherentes, lo que permite la categorización esencialista a partir de ciertas dimensiones como la orientación religiosa, la ideología política, la nacionalidad, etc.

La diferenciación aquí establecida entre ambas formas de esencialización nos permite concluir que el pensamiento esencialista puede ser interpretado como un sesgo heurístico que ofrece ciertos automatismos necesarios para que podamos vivir en un mundo cognitivamente complejo, al tiempo que facilita la preservación de las diferencias de poder y mantiene las desigualdades sociales, políticas y económicas, al definir las categorías sociales como algo inmutable (Mahalingam, 2003). Así caracterizado, el esencialismo puede ser interpretado como una forma de categorización social que asume la tesis de que las diferentes manifestaciones de la identidad social pueden ser esencializadas en varios niveles, dependiendo de los propósitos establecidos por los distintos grupos humanos (Gelman, 2004; Haslam, Bain, Douge, Lee y Bastian, 2005; Haslam, Bastian y Bisset, 2004; Keller, 2005; Picket, 2001).

Esta discusión acerca del esencialismo es pertinente para el estudio de la categorización realizada en función del género por varios motivos, entre los que cabe destacar dos de ellos. En primer lugar, es importante considerar que la división de los papeles sociales continúa estando marcada más por las categorías naturalistas hombre-mujer que por las categorías entitativas masculino-femenino. Las creencias estereotipadas tienen un efecto en la naturalización de las diferencias entre hombres y mujeres, pues a los primeros se les atribuyen características como el poder y el control de recursos, además de ser percibidos como más racionales, independientes, asertivos, competitivos y ambiciosos, en cuanto que a las mujeres se les atribuyen rasgos como la dependencia, la sensualidad, la sensibilidad, la protección, la afectividad y la sumisión. En segundo lugar, los papeles sociales tradicionalmente realizados por hombres y mujeres favorecen la formación de estereotipos de género, al tiempo que 
presuponen y justifican las diferencias de poder en las relaciones de género (Assmar y Ferreira, 2004).

En líneas generales, prevalece la creencia estereotipada de que los hombres están dotados de características personales entre las que sobresalen la acción y la instrumentalidad, mientras que las mujeres son percibidas como más emocionales y volcadas a los cuidados de la prole y a las tareas requeridas para el buen funcionamiento de la familia y el hogar (Eagly, 1987). Si bien es cierto que los cambios sociales ocurridos en las últimas décadas, debidos principalmente a la inserción de las mujeres en el mercado de trabajo - o sea, en la esfera de lo público - han hecho que las diferencias en las ocupaciones ejercidas por hombres y mujeres sean más difusas, sigue siendo común que hombres y mujeres sean juzgados de acuerdo a dos dimensiones independientes: la cordialidad, asociada al afecto, una característica estereotípicamente asociada a las mujeres y la competencia, atribuida, en general, a los hombres (Fiske, 2012; Fiske, Cuddy, Glick y Xu, 2002).

Finalmente, es importante señalar que las cuestiones suscitadas en los estudios sobre las relaciones entre géneros no son meramente teóricas. Las perspectivas construccionistas y feministas están arraigando en las sociedades contemporáneas (Saavedra y Nogueira, 2006). El papel de las creencias esencialistas representa una de las dimensiones más importantes no sólo en el plano teórico, sino también en la confrontación que se expresa en la cultura popular mediante la expresión: "guerra de sexos". La popularización de este debate hace que la cuestión que anima este artículo sea de mayor interés: ¿qué hace que el raciocinio esencialista sobreviva con facilidad en un medio en el cual las visiones antiesencialistas desempeñan un importante papel en la lucha contra las desigualdades entre hombres y mujeres?

\section{Objetivos e hipótesis}

Este estudio da cuenta de los resultados de un estudio experimental realizado en España y en Brasil y basado en el denominado paradigma del trasplante de cerebros de Mahalingam (2003). Este autor, al analizar el impacto del esencialismo en la sociedad de castas en la India, distingue tres formas de esencialismo; la primera de carácter biológico, en la cual la esencialización estaría basada en argumentos fundamentados en la biología y en la genética, una segunda que estaría basada en argumentos sociales, en donde la categorización sería interpretada como una consecuencia del proceso de socialización y, por último, un tercer tipo de esencialización que estaría basado en argumentos referidos a la influencia de lo transcendental o sobrenatural, en el que la esencialización sería elaborada a partir de la referencia a vidas pasadas. Aunque no sea adecuado trazar paralelismos entre culturas tan diferentes como la referida en la investigación de Mahalighan y las del estudio aquí presentado, nos interesa destacar que el esencialismo se nutre de un conjunto amplio de heurísticos y argumentos con los que explicar las diferencias sociales entre los grupos. En este estudio partimos del presupuesto de que la adopción del raciocinio esencialista en las relaciones de género está influido por circunstancias sociales y culturales que procuramos evaluar teniendo en cuenta su presencia en dos contextos sociales diferenciados. Asimismo, estudiamos el tipo de explicaciones que los participantes dan sobre la inmutabilidad o el cambio en el comportamiento de hombres y mujeres. La elección de la categoría género está basada en estudios previos como el de Haslam, Rothschild y Ernst (2000) quienes encontraron que el género es la categoría social más asociada a las propiedades de las categorías naturales (naturalidad, necesidad, inmutabilidad, discrecionalidad y estabilidad). Asimismo, el género es entre todas las categorías sociales la que está más fuertemente asociada al raciocinio esencialista desde la infancia, teniendo un marcado potencial inductivo (Prentice y Miller, 2006).

El objetivo fundamental de este trabajo de investigación fue evaluar si ante un supuesto trasplante de cerebro entre un hombre y una mujer se observarían cambios en la conducta del receptor de dicho trasplante. El estudio tuvo en cuenta tanto el impacto del país, Brasil y España, como la dirección del trasplante (cuando el cerebro de un hombre era trasplantado a una mujer y a la inversa). Finalmente, se analizaron las justificaciones que los participantes en el estudio dieron para el cambio o estabilidad en la conducta del supuesto receptor del trasplante. Los resultados expuestos en este trabajo se refieren a las siguientes hipótesis:

H1) En relación a la versión naturalista del esencialismo, se espera una mayor esencialización de las categorías naturalizables, como el género (hombre-mujer), la edad (adultoniño) o la raza (blanco-negro), si las comparamos con las categorías entitativas, formadas por la orientación religiosa (católico-musulmán), o por diferencias económicas (ricopobre), sociales (policía-delincuente), políticas (derechaizquierda) y nacionales (nacional-extranjero).

H2) En relación a la esencialización realizada a través de la entitatividad y considerando las diferencias históricas y culturales, así como las diferencias en el grado de organización de los movimientos feministas, se esperan diferencias significativas en las respuestas de los participantes brasileños y españoles sobre los cambios en la conducta resultado del supuesto trasplante de cerebros.

H3) Dado el predominio de una visión androcéntrica del mundo, se esperan diferencias en la dirección del trasplante de cerebro, en concreto una mayor estabilidad en la conducta de los hombres después de recibir el supuesto trasplante de una mujer y una mayor influencia en el cambio de conducta cuando el cerebro de un hombre es supuestamente trasplantado a una mujer.

H4) Considerando las diferentes teorías implícitas posibles para la explicación de las respuestas de los participantes a los posibles efectos del trasplante en la conducta, se espera un predominio de explicaciones basadas en las causas internas en detrimento de las teorías explicativas fundamentadas en la intencionalidad. 


\section{Método}

Se trata de un estudio experimental destinado a evaluar la influencia del país y de la dirección del supuesto trasplante en los procesos de esencialización de diferentes categorías sociales, especialmente entre hombres y mujeres.

\section{Participantes}

Los datos obtenidos en España se refieren a una muestra de 138 estudiantes y fueron recogidos en la Universidad Complutense de Madrid, en las Facultades de Ciencias Políticas y Sociología y en la Facultad de Ciencias Biológicas. En Brasil, de un total de 101 participantes, 66 eran estudiantes de la Escuela de Administración y de la Facultad de Derecho de la Universidad Federal de Bahía y 35 eran estudiantes de Psicología y Ciencias Biológicas de la Universidad Presidente Antônio Carlos, de la ciudad de Barbacena, en el Estado de Minas Gerais.

\section{Procedimientos}

En esta investigación se adoptó un procedimiento de investigación que sigue los protocolos experimentales realizados por Mahalingam (2003) para la evaluación de la esencialización entre brahmanes y dablits en la India. Este paradigma experimental tiene su origen en la psicología del desarrollo, habiendo sido utilizado originalmente para investigar la estabilidad en la identidad personal (Gelman, 2004; Gottfried, Gelman y Schultz, 1999; Johnson, 1990).

Los participantes fueron asignados aleatoriamente a dos grupos. Debían leer historias breves sobre un hipotético personaje que recibía un trasplante de cerebro de un individuo que pertenecía a una categoría a la que él o ella no pertenecían. El enunciado de estas historias se encuentra ejemplificado a continuación y representa la versión en la cual el cerebro de una categoría hegemónica o dominante (hombre) es trasplantado a una persona perteneciente a una categoría social no hegemónica o no dominante (mujer).

Alguien trasplanta el cerebro de María y lo coloca en la cabeza de Pablo. Después de este trasplante, como piensa usted que se comportará Pablo, como:

a) hombre

b) mujer

Cada participante evaluó ocho historias, tres referentes a categorías más naturalizables (género, edad y raza) y cinco a categorías más entitativas (nacionalidad, orientación política, creencias religiosas, posición económica y estatus social). Para cada una de las ocho versiones fueron presentados dos tipos de historias, siendo una versión relativa al trasplante de cerebros de miembros de categorías hegemónicas para las no hegemónicas (hombre para mujer; adulto para niño; blanco para negro; rico para pobre; etc) y la otra del trasplante de cerebros de miembros de categorías no hegemónicas a categorías hegemónicas (mujer para hombre, niño para adulto, negro para blanco, pobre para rico; etc). Cada participante evaluó exclusivamente una de las dos versiones, bien de la categoría hegemónica para la no hegemónica o la opuesta, en relación a cada una de las ocho categorías sociales. Todo el procedimiento de recogida de datos fue realizado mediante el uso de un protocolo computerizado que permitió estandarizar la presentación de las instrucciones, aleatorizar el orden de presentación de los ítems, reproducir las respuestas a los ítems, solicitar las justificaciones para las respuestas, medir las respuestas, ofrecer a cada participante un feedback sobre las respuestas y generar una base de dados.

\section{Instrumentos}

La recogida de datos fue enteramente realizada mediante el uso de un dispositivo computacional, creado con el sistema Toolbook Instructor, versión 5.01, especialmente diseñado para esta investigación. Mediante algoritmos computacionales, fue posible aleatorizar el orden de presentación de las categorías, así como estandarizar las instrucciones para los participantes. La traducción y adaptación del instrumento, originalmente creado en portugués fue realizada por nativos bilingües.

\section{Diseño}

El diseño experimental adoptado en el presente estudio corresponde al modelo 2 (Brasil, España) x 2 (dirección de trasplante de cerebro) x 2 (grupos naturalizables, grupos entitativos), siendo los dos primeros factores independientes y el último de medidas repetidas.

Una serie de estrategias distintas fueron adoptadas para el análisis de los datos. Una vez recogidas las respuestas nominales de cada participante a cada una de las historias presentadas, se elaboraron distintas tablas de contingencia con el objetivo de identificar el grado de asociación entre las respuestas a cada una de las variables independientes. Para la elaboración de las tablas de contingencia, dichas respuestas fueron codificadas como 0 , no habrá cambio en la conducta y 1, habrá cambio en la conducta. Así, cuando el participante indicó que Pablo se comportaría como una mujer tras el trasplante, la respuesta era codificada como 1; en el caso de que afirmase, por el contrario, que Pablo continuaría comportándose como hombre, la respuesta era codificada como 0.

Para poner a prueba nuestras hipótesis fueron construidos diversos índices basados en las respuestas dadas a las categorías naturalizables y entitativas. El primero de estos índices corresponde a la media de las respuestas dadas a los ítems relativos a cada una de las ocho categorías, cuyos valores de respuesta oscilaron en un rango que iba de 0 , cuando todas las categorías fueron esencializadas, a 8 , que indicaba que ninguna de las categorías fue esencializada. De esta misma forma, fue elaborado un índice para el cálculo de las categorías naturalizables (sexo, edad, raza) y otro para las categorías entitativas (religión, ideología política, etc). Los 
valores de estos índices fueron utilizados como variables dependientes para comparar las diferencias entre el grado de naturalización y de entitatividad mediante el test de la $t$ de medidas repetidas. Estos análisis fueron complementados con la realización de un análisis log-linear para cada una de las variables, lo que permitió conocer el impacto conjunto del país y la dirección del trasplante de cerebro en la manifestación del pensamiento esencialista.

\section{Análisis y discusión de los resultados}

Si bien el presente estudio tiene por objetivo principal presentar y discutir los datos obtenidos con participantes brasileños y españoles relativos a la categoría naturalista hombremujer, antes es necesario situar estos resultados integrándolos en un contexto más amplio del cual esta investigación forma parte, por lo que para la comparación entre el grupo de las categorías naturalizables y las entitativas, en su conjunto, haremos referencia a la muestra inicial de esta investigación (Pereira, Álvaro y Schweiger, 2010).

El objetivo central de la misma fue el de someter a prueba la hipótesis de que las categorías naturalistas serían más esencializadas que las categorías entitativas, conforme a la H1. Un test de la $t$ entre las medias de las categorías sociales naturalizables y entitativas evidenció una diferencia significativa $\left(t_{(266)}=5.61, p<.001\right)$, en el sentido que se identificó una esencialización más intensa para las categorías naturalizables (género, edad y raza), si las comparamos con las categorías entitativas establecidas en función de la orientación religiosa (católico-musulmán), diferencias económicas (ricopobre), sociales (policía-delincuente), políticas (derechaizquierda) y nacionales (nacional-extranjero).

Si bien la media de las categorías naturalizables presenta un mayor potencial para la esencialización que las categorías entitativas, es importante describir las diferencias observadas en los resultados de los ítems asignados a una misma categoría. Si consideramos los primeros valores de la columna relativa a la estabilidad de la conducta en la Tabla 1, que es un indicador del esencialismo por vía de la naturalización, es evidente que aunque la categoría género no sea tan esencializada como la categoría raza, sufre un proceso de esencialización mucho más acentuado que la categoría edad. Los resultados relativos a las dos primeras categorías se contraponen claramente a los encontrados para las categorías entitativas, excepto la de rico-pobre, cuyos valores se asemejan a los identificados para las categorías naturalizables.

De modo general, los resultados obtenidos son consistentes con la $\mathrm{H} 1$, evidenciando la creencia de que si un cerebro trasplantado lleva en sí las características del donante, tales características acabarán ejerciendo una influencia sobre la conducta del receptor. Este efecto no ocurre de forma homogénea en todas las categorías sociales, al ser menos acentuado en las categorías entitativas que en las categorías naturalistas. Rotular a alguien como blanco o negro, hombre o mujer, parece inhibir el potencial del cerebro trasplantado de modificar la conducta del receptor cuando lo comparamos con la utilización de rótulos como derecha e izquierda o policía y delincuente.

Tabla 1. Frecuencia y porcentaje de estabilidad y cambio de las categorías naturalistas y entitativas.

\begin{tabular}{lcccc}
\hline Categorías & \multicolumn{2}{c}{ Cambio } & \multicolumn{2}{c}{ Estabilidad } \\
\cline { 2 - 5 } & $F$ & $\%$ & $F$ & $\%$ \\
\hline Naturalistas & 118 & 50.9 & 114 & 49.1 \\
$\quad$ Raza (blanco-negro) & 140 & 60.3 & 92 & 39.7 \\
$\quad$ Sexo (hombre-mujer) & 156 & 67.2 & 76 & 32.8 \\
$\quad$ Edad (adulto-niño) & & & & \\
Entitativas & 122 & 52.6 & 110 & 47.4 \\
$\quad$ Económica (rico-pobre) & 157 & 67.7 & 75 & 32.3 \\
Nacional (nativo-extranjero) & 163 & 70.3 & 69 & 29.7 \\
$\quad$ Religión (católico-musulmán) & 171 & 73.7 & 61 & 26.3 \\
$\quad$ Social (policía-delincuente) & 189 & 81.5 & 43 & 18.5 \\
$\quad$ Política (derecha-izquierda) & & & & \\
\hline
\end{tabular}

Una segunda cuestión que este estudio pretende analizar es si los efectos observados se darán de la misma forma en los dos países en los cuales el estudio fue realizado. Finalmente, se estudió si la dirección del trasplante, tendría, a su vez efectos diferenciados

Para poner a prueba las hipótesis $\mathrm{H} 2$ y $\mathrm{H} 3$ fue realizado un análisis loglinear, mediante el uso del procedimiento por eliminación backward stepwise, que generó un modelo (likelihood ratio $x^{25}=2.87, p=.412$ ) en el que se identificaron dos tipos de interacción, la primera entre el país y el tipo de repuestas y la segunda entre el país y la dirección del trasplante ${ }^{1}$.

El análisis de los valores relativos a los coeficientes logarítmicos de proporcionalidad presentados en la tabla 2 permite identificar una mayor esencialización entre los participantes brasileños. Es importante señalar que un valor positivo del coeficiente de proporcionalidad logarítmico indica un predominio de las respuestas de estabilidad sobre las de cambio en la conducta, o sea, una mayor esencialización. Conforme se observa en la última línea de la tabla, la única condición en la que se obtiene un coeficiente logarítmico positivo, de .16, fue la referida a los participantes brasileños y se corresponde con el trasplante de cerebro de una mujer para un hombre. Hay que destacar que incluso en el caso de los participantes brasileños, el valor -.04 no permite afirmar la existencia de esencialización y que en el caso de los participantes españoles, las puntuaciones obtenidas, ambas negativas de -.11 y -.35 , indican respuestas no esencializadoras.

\footnotetext{
${ }^{1}$ Se realizó también un análisis log-lineal de tres vías, incluyendo el género como criterio diferenciador, que no ofreció cambios significativos a los aquí presentados.

Estos valores son compatibles no solamente con la bidireccionalidad inherente a la hipótesis $\mathrm{H} 2$, sino que también indican respuestas consistentes con la hipótesis de la mayor esencialización entre los participantes brasileños que entre los españoles. Tal y como fue planteado anteriormente, dla dirección del trasplante de cerebros, de una categoría hegemónica para una no hegemónica ejerce alguna influencia en los resultados?
} 
Tabla 2. Porcentajes, frecuencias obtenidas y esperadas, coeficientes de proporcionalidad y coeficientes logarítmicos de las respuestas de estabilidad y cambio de la conducta como consecuencia de un supuesto trasplante de cerebros entre hombres y mujeres.

\begin{tabular}{|c|c|c|c|c|c|c|c|c|c|}
\hline \multirow{3}{*}{$\begin{array}{l}\text { País } \\
\text { Dirección Conducta }\end{array}$} & & \multicolumn{4}{|c|}{ España } & \multicolumn{4}{|c|}{ Brasil } \\
\hline & & \multicolumn{2}{|c|}{ Varón - Mujer } & \multicolumn{2}{|c|}{ Mujer - Varón } & \multicolumn{2}{|c|}{ Varón - Mujer } & \multicolumn{2}{|c|}{ Mujer - Varón } \\
\hline & & Estabilidad & Cambio & Estabilidad & Cambio & Estabilidad & Cambio & Estabilidad & Cambio \\
\hline Porcentaje & & 43.4 & 56.6 & 30.8 & 69.2 & 49.0 & 51.0 & 54.0 & 46.0 \\
\hline \multicolumn{10}{|l|}{ Frecuencias } \\
\hline & Obtenidas & 33 & 43 & 20 & 45 & 25 & 26 & 27 & 23 \\
\hline & Esperada final & 30.1 & 45.9 & 25.8 & 39.2 & 26.3 & 24.7 & 25.7 & 24.3 \\
\hline OR P x D X R & & \multicolumn{2}{|c|}{.77} & \multicolumn{2}{|c|}{.44} & \multicolumn{2}{|c|}{.96} & \multicolumn{2}{|c|}{1.17} \\
\hline OR $\log \mathrm{P} \times \mathrm{D} \times \mathrm{R}$ & & \multicolumn{2}{|c|}{-.11} & \multicolumn{2}{|c|}{-.35} & \multicolumn{2}{|c|}{-.04} & \multicolumn{2}{|c|}{.16} \\
\hline
\end{tabular}

A partir del supuesto de que las categorías hegemónicas se asocian a un potencial de estabilidad en la conducta, formulamos la hipótesis $\mathrm{H} 3$, en la que postulamos una mayor estabilidad en la conducta de los hombres después del trasplante de cerebro, lo que implica también un mayor poder de influencia del cerebro masculino sobre la conducta de la mujer como receptora del trasplante. El análisis de las frecuencias esperadas y obtenidas permite identificar que la influencia de la dirección del trasplante de cerebros se manifiesta de forma diferenciada entre los participantes españoles y brasileños. En el caso de los participantes españoles, éstos consideran que la mujer tiene mayores probabilidades de mantener su conducta estable después de recibir el cerebro de un hombre $(33.0 \times 30.1)$ y, en contrapartida, que la conducta de un hombre tiene más probabilidades de sufrir cambios en el caso de recibir el cerebro de una mujer $(20.0 \mathrm{x}$ 25.8). Entre los participantes españoles queda claro un índice menor de estabilidad cuando el trasplante es realizado desde una categoría menos hegemónica a una hegemónica, lo que contradice la hipótesis inicialmente formulada. Entre los participantes brasileños, los resultados presentan una tendencia inversa, compatible con la hipótesis H3, si bien las diferencias entre los valores esperados y obtenidos fueron bastante menos acentuadas que en el caso de las diferencias observadas en la muestra española.

Al contrario que las hipótesis anteriores, cuyos análisis fueron elaborados a partir de la respuesta a la pregunta sobre si el trasplante provocaría o no algún cambio en la conducta del receptor del cerebro trasplantado, para poner a prueba la hipótesis $\mathrm{H} 4$ se analizaron los argumentos y justificaciones presentadas por los propios participantes a sus respuestas. Esperábamos un mayor número de justificaciones relacionadas con causas internas frente a otros tipos de explicación. Con el fin de contextualizar los resultados obtenidos y facilitar la comparación con el resto de categorías que fueron objeto de análisis inicial en el estudio más amplio que dio origen al presente estudio, presentamos en la tabla 3 los resultados relativos a las ocho categorías sociales, tres naturalizables y cinco entitativas ya descritas con anterioridad. El criterio adoptado para la clasificación de las respuestas fue el modelo folk de explicación de las conductas sociales desarrollado por Malle (1999; 2006), en el que se postulan cuatro modalidades de teorías explicativas de la conducta: las causas internas, la historia causal, los factores habilitadores y, finalmente, los motivos y razones.

Conforme se observa en los valores de los coeficientes de proporcionalidad logarítmicos, los resultados generales dejan claro que las teorías explicativas fundamentadas en los factores habilitadores, aquí denominados presiones situacionales (.51), fueron adoptadas preferentemente para explicar la estabilidad en la conducta. Las demás teorías explicativas presentan coeficientes de proporcionalidad logarítmicos negativos, -.58 para las causas internas, -.47 para la historia causal y - .51 para los motivos y razones, resultados que pueden ser interpretados como indicadores de respuestas de cambio en la conducta subsecuentes al presunto trasplante de cerebros.

Las causas internas representan la principal fuente de explicación responsable por casi un $60 \%$ de las justificaciones, seguida por la historia causal, que fue adoptada como justificación en un $24 \%$ de los casos. Las presiones situacionales, aunque predominantes en la explicación de la estabilidad en la conducta, fueron responsables por apenas un $15 \%$ de las explicaciones globales. Es importante señalar que el escasísimo número de explicaciones fundamentadas en las razones, responsables por apenas 17 de un total de 1551 justificaciones, probablemente sea debido al contenido de la tarea experimental incluida este estudio, que no favorece la adopción de explicaciones fundamentadas en la intencionalidad.

Las justificaciones presentadas para las respuestas relativas a la categoría sexo en cierta medida confirman la tendencia general. La más intencional de las fuentes de explicación, los motivos y las razones no fueron mencionados por ninguno de los participantes. Las causas internas fueron las más aludidas, alcanzando cerca del $77 \%$ de todas las explicaciones, habiendo sido adoptadas prioritariamente para la explicación de los cambios en la conducta $(56 \times 97)$. La historia causal fue mencionada en un $18 \%$ de las explicaciones, habiendo sido utilizada prioritariamente para la explicación de los cambios en la conducta $(11 \times 24)$. Las presiones situacionales, aunque en una proporción menor que en el resto de categorías, fueron adoptadas, prioritariamente, para la explicación de la estabilidad en la conducta $(5 \times 4)$. 
Tabla 3. Frecuencias de las justificaciones presentadas, clasificadas según los tipos de explicación.

\begin{tabular}{|c|c|c|c|c|c|c|c|c|c|}
\hline (2) & \multicolumn{2}{|c|}{$\begin{array}{c}\text { Causas internas } \\
\text { Genes, hormonas, cerebro, } \\
\text { cuerpo, rasgos y procesos } \\
\text { psicológicos, esencias, self }\end{array}$} & \multicolumn{2}{|c|}{$\begin{array}{c}\text { Historia causal } \\
\text { Creencias, valores, actitude } \\
\text { apariencia común }\end{array}$} & \multicolumn{2}{|c|}{$\begin{array}{c}\text { Presiones situacionales } \\
\text { Estatus, roles, dinámica grupal } \\
\text { y colectiva, ambiente social }\end{array}$} & \multicolumn{2}{|c|}{$\begin{array}{l}\text { Razones } \\
\text { Motivos e } \\
\text { intenciones }\end{array}$} & \multirow[t]{2}{*}{ Total } \\
\hline & Estabilidad & Cambio & Estabilidad & Cambio & Estabilidad & Cambio & Estabilidad & Cambio & \\
\hline Raza & 45 & 46 & 13 & 41 & 15 & 1 & 0 & 1 & 162 \\
\hline Edad & 40 & 112 & 14 & 33 & 9 & 4 & 0 & 0 & 212 \\
\hline Sexo & 56 & 97 & 11 & 24 & 5 & 4 & 0 & 0 & 197 \\
\hline Economía & 9 & 60 & 6 & 22 & 64 & 23 & 0 & 0 & 184 \\
\hline Política & 9 & 133 & 11 & 27 & 16 & 3 & 0 & 0 & 199 \\
\hline Nacionalidad & 8 & 80 & 16 & 54 & 20 & 8 & 0 & 0 & 186 \\
\hline Religión & 14 & 92 & 19 & 48 & 25 & 7 & 1 & 4 & 210 \\
\hline Social & 10 & 111 & 5 & 31 & 27 & 6 & 3 & 8 & 201 \\
\hline Subtotal & 191 & 731 & 95 & 280 & 181 & 56 & 4 & 13 & \\
\hline Total (\%) & \multicolumn{2}{|c|}{$922(60 \%)$} & \multicolumn{2}{|c|}{$375(24 \%)$} & \multicolumn{2}{|c|}{$237(15 \%)$} & \multicolumn{2}{|c|}{$17(1 \%)$} & 1551 \\
\hline Odds ratio & \multicolumn{2}{|c|}{.26} & \multicolumn{2}{|c|}{.35} & \multicolumn{2}{|c|}{3.23} & \multicolumn{2}{|c|}{.31} & \\
\hline Odds ratio log & \multicolumn{2}{|c|}{-.58} & \multicolumn{2}{|c|}{-.47} & \multicolumn{2}{|c|}{.51} & \multicolumn{2}{|c|}{-.51} & \\
\hline
\end{tabular}

Las explicaciones presentadas por los participantes, en el caso específico de las causas internas, estuvieron basadas en causas anatómicas y fisiológicas, siendo especialmente significativa la referencia a argumentos elaborados en torno al concepto de hormonas ("el cerebro es de hombre, pero el cuerpo es de mujer y las hormonas femeninas terminarán variando su anterior conducta de hombre en mujer", caso 26, estabilidad, España; "El cambio en la forma de pensar no sólo viene por las vivencias establecidas ya en el cerebro sino también por regulación hormonal', caso 76, cambio, España). Además de las hormonas, otras fuentes de explicación interna de la conducta se refieren a argumentos biológicos, relacionados con factores genéticos ("porque el cerebro sólo tiene información y conocimiento y el hecho de ser hombre o mujer va en los genes y no en el cerebro o la inteligencia", caso 22, estabilidad, España), los órganos sexuales ("en la determinación del sexo juegan un papel muy importante los órganos sexuales, que interactúan con el cerebro", caso 134, cambio, España; o el propio cerebro ("el cerebro de Pablo se ha desarrollado para el sexo masculino, por lo que presentará estimuladas aquellas características cerebrales presentes en un hombre, y las conservará en María", caso 33, cambio, España; "el cerebro es el principal órgano donde se elaboran las órdenes", caso 106, cambio, España. Las causas internas, sin embargo, no se restringen a los factores biológicos, ya que podemos identificar una serie de justificaciones basadas en factores psicológicos como los rasgos de personalidad ("las mujeres tienen unos rasgos psíquicos distintos de los hombres que no se pueden ignorar", caso 137, cambio, España) o incluso los instintos ("porque pienso que los instintos permanecen", caso 225, estabilidad, Brasil).

Otro tipo de explicación reside en la historia causal de las razones, adoptada particularmente para justificar los cambios en la conducta. Las principales fuentes de explicación dentro de esta categoría incluyen el proceso de socialización ("el género no está en el cerebro sino en los procesos de socialización", caso 79, estabilidad, España; "hay diferencias conductuales entre hombres y mujeres y éstas son aprendidas en la infancia", caso 53, cambio, España), el estilo de vida ("será el de un hombre porque siempre ha vivido como tal y su estilo de vida está condicionado por su sexo", caso
77, estabilidad, España), la cultura ("María continuará comportándose como mujer, pues ella adquirió la cultura, valores y creencias de mujer", caso 147, estabilidad, Brasil), la sociedad ("el sexo es una construcción social, lo biológico apenas determina la conducta por lo que a pesar de tener un cuerpo de mujer el cerebro de hombre determinará su conducta", caso 62, cambio, España) o la mentalidad ("la mentalidad de ella es femenina y aunque el cuerpo pase a ser de hombre, continuará actuando como mujer", caso 121, cambio, Brasil; "la visión del mundo ya estará construida", caso 193, cambio, Brasil).

En contraposición a los argumentos anteriores, utilizados prioritariamente para la explicación de los cambios en la conducta después del trasplante entre personas de distinto sexo, las presiones situacionales fueron adoptadas para justificar la estabilidad de la conducta. La respuesta predominante se refirió a la influencia del medio ambiente ("porque su entorno sigue siendo estable, por lo que reaccionará según éste se encuentre", caso 109, estabilidad, España; "la conducta está determinada por experiencias previas, después podría variar por el nuevo entorno", caso 75, cambio, España), aunque también encontramos justificaciones que se refieren a factores tan dispares como las contingencias de la vida ("aunque con el tiempo necesitará mantener relaciones sexuales, eso le hará convertirse en mujer definitivamente", caso 43, cambio, España) los roles sociales ("Hay distinción de género y de sexo en la sociedad, y desempeñaria el rol femenino", caso 119, cambio, España) e incluso, la sociedad ("esto es un hecho social. Si él tiene cuerpo de hombre y actuaba como tal, la sociedad le "exigirá" que se mantenga como tal", caso 182, estabilidad, Brasil).

\section{Conclusiones}

Los resultados presentados, considerados en su conjunto, permiten describir un cuadro general sobre el proceso de esencialización de hombres y mujeres. Como se trata de una categoría más naturalizable, el grado de esencialización de la categoría hombre-mujer fue más intenso que el observado en el caso de las categorías entitativas, con excepción de la 
categoría pobre-rico. En líneas generales, fue posible identificar la influencia del país en el tipo de respuestas, lo que nos permite observar un menor grado de esencialización entre los participantes españoles que entre los brasileños.

¿Qué podría explicar esta diferencia en los resultados relativos a hombres y mujeres? Si consideramos los resultados obtenidos en los análisis de las hipótesis $\mathrm{H} 2$ y H3, los participantes brasileños tienden a esencializar de forma más indiscriminada, mientras que la utilización del esencialismo resulta un proceso más complejo entre los participantes españoles, pues está sometido a la influencia directa de la dirección del supuesto trasplante; una tendencia que no se observa entre los participantes brasileños. En el caso de los participantes españoles el trasplante tanto en hombres como en mujeres puede influir en el cambio de conducta de las personas del otro sexo, si bien esta tendencia es mayor cuando el receptor del referido trasplante de cerebro es un hombre. Sin embargo, en el caso de la muestra brasileña, los resultados indican una mayor estabilidad en la conducta tras el supuesto trasplante, lo que es compatible con la tesis de que tanto hombres como mujeres tienen patrones de conducta que por su propia naturaleza son más estables y poco sujetos al cambio.

Estos resultados, en su conjunto, pueden ser interpretados de dos maneras. Una primera explicación es que son el reflejo de relaciones de género menos igualitarias entre los participantes brasileños si las comparamos con las que encontramos entre los españoles. Otra posible explicación sería que los datos ponen en evidencia una menor esencialización naturalista entre los participantes españoles. Los procesos de categorización aquí descritos probablemente deben tomar en consideración una interpretación en la que los componentes biológicos y sociales establecen varias modalidades de interacción, en la cuales los primeros reproducen las diferencias tradicionales entre los sexos y los segundos determinan los cambios sociales que ocurren en la representación social de las diferencias de género (DeCecco y Elia, 1993). Adicio-

\section{Referencias}

Allport, G. (1962). La naturaleza del prejuicio. Buenos Aires: Eudeba.

Anderson, E. (1995). Feminist Epistemology: An Interpretation and Defense. Hypatia, 10, 50-84. doi: 10.1111/j.1527-2001.1995.tb00737.x Assmar, E. y Ferreira, M. C. (2004). Estereótipos e preconceitos de gênero, liderança e justiça organizacional: controvérsias e sugestões para uma agenda de pesquisa. En M. E. Lima y M. E. Pereira (Eds.), Estereótipos, preconceitos e discriminação (pp. 89-116). Salvador: Edufba.

Campbell, D. (1958). Common fate, similarity, and other indices of the status of aggregates of persons as social entities. Behavioral Science, 3, $14-25$.

DeCecco, J. y Elia, J. (1993). Critique and synthesis of biological essentialism and social constructionist views of sexuality and gender. Journal of Homosexuality, 24(3-4), 1-26. doi: 10.1300/J082v24n03_01

Demoulin, S., Leyens, J.Ph. y Yzerbyt, V.Y. (2006). Lay theories of essentialism. Group Processes and Intergroup Relations, 9, 25-42. doi:10.1177/1368430206059856

Duran, J. (1991). Toward a Feminist Epistemology. Savage, Md.: Rowman \& Littlefield.

Eagly, A. (1987). Sex differences in social behavior: A social-role interpretation. Hillsdale: Erlbaum. nalmente, debemos insistir en la importancia de las causas internas y de la historia causal como fuentes argumentativas para explicar los cambios en la conducta provocados por el supuesto trasplante.

Los resultados de este estudio indican la necesidad de una reflexión sobre los propios fundamentos de las teorías esencialistas, pues la esencialización no se restringe ni se limita a los argumentos que se centran en la explicación de la estabilidad de la conducta. Los estudios fundamentados en la idea de un trasplante de cerebro entre diversas categorías sociales permiten, al menos hipotéticamente, sugerir la posible existencia de una creencia en que las esencias pueden ser transferidas y que la transferencia de estos atributos esencializadores puede proporcionar patrones de conducta que no pueden ser explicados mediante la utilización de argumentos elaborados a partir de las clásicas nociones de estabilidad e invariabilidad de la conducta asociadas a los procesos de esencialización de categorías naturalizables.

En definitiva, las implicaciones que estos resultados ofrecen son importantes para la comprensión de los estereotipos de género, particularmente porque nos indican la necesidad de ser entendidos de forma más profunda, poniendo en relación las teorías sobre la naturalización, tradicionalmente transmitidas por medio de la educación familiar y escolar y las teorías sobre el género elaboradas en el seno de las epistemologías feministas. Este estudio deja claro que, potencialmente, las "esencias" no sólo sirven para naturalizar las conductas de ciertas categorías sociales, sino que pueden ser transferidas provocando cambios en la conducta. Este dato supone nuevos desafíos para los que se dedican a la tarea de combatir los negativos efectos de los estereotipos de género, pues si se confirma la existencia de creencias basadas en la idea de que las esencias pueden ser transferidas entre individuos de sexos diferentes, intentar cambiar los rasgos de personalidad y las características estables de ciertas categorías sociales puede ser una medida menos efectiva de lo que pensamos.

Fine, C. (2010). Delusions of gender: How our minds, society, and neurosexism create difference. Nueva York: Norton.

Fiske, S. T. (2012). Warmth and competence: Stereotype content issues for clinicians and researchers. Canadian Psychology/Psychologie Canadienne, 53(1), 14-20. doi: 10.1037/a0026054

Fiske, S., Cuddy, A., Glick, P. y Xu, J. (2002). A Model of (Often Mixed) Stereotype Content: Competence and Warmth. Respectively Follow From Perceived Status and Competition. Journal of Personality and Social Psychology, 82(6), 878-902. doi: 10.1037/0022-3514.82.6.878

Gelman, S. A. (2004). Psychological essentialism in children. Trends in Cognitive Sciences, 8, 404-409. doi: 10.1016/j.tics.2004.07.001

Gelman, S. y Wellman, W. (1991). Insides and essences: early understandings insides and essences of the non-obvious. Cognition, 38, 213-244. doi: 10.1016/0010-0277(91)90007-Q

Gottfried, G. M., Gelman, S. A. y Schultz, J. (1999). Children's understanding of the brain: From early essentialism to biological theory. Cognitive Development, 14, 147-174. doi: 10.1016/S0885-2014(99)80022-7

Haslam, N., Rothschild, L. y Ernst, D. (2000). Essentialist beliefs about social categories. British Journal of Social Psychology, 39: 113-127. doi: 10.1348/014466600164363 
Haslam, N., Bastian, B. y Bisset, M. (2004). Essentialist beliefs about personality and their implications. Personality and Social Psychology Bulletin, 30 , 1661-1673. doi: 10.1177/0146167204271182

Haslam, N., Bain, P., Douge, M., Lee M. y Bastian, B. (2005). More human than you: Attributing humanness to self and others. Journal of Personality and Social Psychology, 89(6), 937-950. doi:10.1037/0022-3514.89.6.937

Johnson, N. C. (1990). If you had my brain, where would I be? Children's understanding of the brain and identity. Child Development, 61, 962-972. doi: 10.1111/j.1467-8624.1990.tb02834.x

Keller, J. (2005). In genes we trust: the biological component of psychological essentialism and its relationship to mechanisms of motivated social cognition. Journal of Personality and Social Psychology, 88(4), 686-702. doi: 10.1037/0022-3514.88.4.686

Mahalingam, R. (2003). Essentialism, culture, and power: representations of social class. Journal of Social Issues, 59(4), 733-749. doi: 10.1046/j.00224537.2003.00087.x

Malle, B. (1999). How people explain behavior: a new theoretical framework. Personality and Psychology Social Review, 3, 23-48. doi: 10.1207/s15327957pspr0301_2

Malle, B. (2006). How the mind explains behavior. Folk explanations, meaning and social interactions. Cambridge, Mass: MIT Press.

Medin, D. (1989). Concepts and conceptual structure. American Psychologist, 44(12), 1469-1481. doi: 10.1037/0003-066X.44.12.1469

Pereira, M. (2002). Psicologia Social dos Estereótipos. São Paulo: E.P.U.
Pereira, M., Álvaro, J.L. y Schweiger, I. (2010). Essentialism and the expression of social stereotypes: a comparative study of Spain, Brasil and England. Spanish Journal of Psychology, 13(2),808-17. doi:10.1017/S1138741600002468.

Pereira, M., Modesto, J. G. y Matos, M. (2013). Em direção a uma nova definição de estereótipos: teste empírico do modelo num primeiro cenário experimental. Psicologia e Saber Social, 1(2), 201-220.

Picket, C. (2001). The effects of entitativity beliefs on implicit comparisons between group members. Personality and Social Psychology Bulletin, 27(5), 515-525. doi: 10.1177/0146167201275001

Prentice, D.A. y Miller, D.T. (2006). Essentializing differences between women and men. Psychological Science, 17,129-135. doi: 10.1111/j.14679280.2006.01675.x

Rothbart, M. y Taylor, M. (1992). Category labels and social reality: Do we view social categories as natural kinds? En G.R.S. Semin y K. Fiedler (Eds), Language, interaction and social cognition (pp. 11-36). Newbury Park, CA: Sage.

Saavedra, L. y Nogueira, C. (2006). Memórias sobre o feminismo na psicologia: para construção de memórias futuras. Memorandum, 11, 113-127.

Yzerbyt, V., Corneille, O. y Estrada, C. (2001). The interplay of subjective essentialism and entitativity in the formation of stereotype. Personality and Social Psychology Review, 5(2), 141-155. doi: 10.1207/S15327957PSPR0502_5

(Articulo recibido: 24-01-2014; revisado: 17-06-2014; aceptado: 15-09-2014) 